\title{
Grupos de alta para pacientes con distimia crónica: una propuesta psicoterapéutica orientada al fin de tratamiento.
}

Groups of discharge for patients with chronic distimia: a psychotherapeutic proposal orientated to the end of treatment.

\author{
Juan Otero Rodríguez ${ }^{\text {a }}$, Aurora Alés Portillo ${ }^{\text {b }}$, Nicolás Vucínovich ${ }^{\mathrm{c}}$. \\ ${ }^{a}$ Psicólogo clínico. ${ }^{b}$ Enfermera especialista en Salud Mental. ${ }^{c}$ Psiquiatra. ${ }^{a, b y c}$ "Unidad de Gestión Clínica \\ de Salud Mental "Virgen del Rocío". Unidad de Salud Mental Comunitaria "Oriente”. Sevilla, España.
}

Correspondencia: Juan Otero Rodríguez(juanotero08@gmail.com)

Recibido: 21/06/2014; aceptado: 23/03/2015

\begin{abstract}
RESUMEN: La intención del autor en este artículo es reflexionar desde la teoría psicoanalítica y desde la experiencia clínica y proponer un planteamiento terapéutico para los pacientes con distimia crónica resistente a los tratamientos. Este trabajo incluye las siguientes formulaciones 1- Los pacientes tratados durante mucho tiempo en las Unidades de Salud Mental Comunitarias y que se cronifican en sus mecanismos patogénicos han de tener una ineludible fecha final de tratamiento 2- Se justifica la psicoterapia de grupo como una posible última fase de su tratamiento en salud mental 3- Se hace la hipótesis de que el alta mejorará la posición de resistencia al cambio psicológico. En este recorrido se revisan los textos freudianos Análisis Terminable e Interminable, Más Allá del Principio de Placer y el concepto de Técnica Activa propuesto por Ferenczi.

A lo largo del artículo se presenta el diseño de los tres grupos terapéuticos realizados, la metodología seguida, los resultados obtenidos, las conclusiones y las preguntas finales.

PALABRAS CLAVE: psicoanálisis, psicoterapia dinámica, psicoterapia de grupo, grupo terapéutico, distimia, alta, fin de tratamiento, Sistema Público de Salud, Unidad de Salud Mental Comunitaria.
\end{abstract}

ABSTRACT: The intention of the author in this article is to think from the psychoanalytic theory and from the clinical experience. and propose a therapeutic plan for patients with distimia chronicle resistants to the treatments. This work includes the following formulations: 1-The patients treated for a long time in the Community Mental Health Units who become chronic in their pathogenic mechanisms have to have an unavoidable final date of treatment 2-we justify the group psychotherapy itself as a last possible phase of the treatment 3it's done the hypothesis that the discharge will improve the position of resistance to a psychological change. We review the Freudian texts: Analysis Terminable and Interminable, Beyond the Pleasure Principle and the concept "active technique" proposed by Ferenczi. Along the article it is presented the design of the three group psychotherapies done, the followed methodology, the obtained results, the conclusions and the final questions.

KEY WORDS: psychoanalysis, dynamic psychotherapy, psychotherapy of group, therapeutic group, distimia, discharge, end of treatment,. Public System of Health, Community Mental Health Unit.

\section{Descripción del perfil de los pacientes}

Este punto describe a los pacientes de los que vamos a hablar.

Los pacientes de los que nos ocuparemos en este artículo son atendidos en una Unidad de Salud Mental Comunitaria. Están diagnosticados de distimia según la clasificación CIE-10. Estos pacientes manifiestan por un periodo mínimo de dos años un variado cortejo de síntomas (tristeza, llanto, anhedonia, apatía, rumiaciones, 
cavilaciones sobre su pasado, ansiedades acerca de su vida presente y futura, insatisfacción, desgana, sensación de impotencia, somatizaciones, problemas para conciliar el sueño, aislamiento, etc.). Estos síntomas les limitan para un funcionamiento cotidiano normal.

Todos ellos llevan un muy largo tiempo de seguimiento en la Unidad de Salud Mental sin que se haya producido una mejoría clínica de sus síntomas ni se hayan modificado sus mecanismos psicológicos disfuncionales. Los síntomas se han perpetuado independientemente del abordaje terapéutico empleado por los profesionales.

\section{El lugar (¿iatrogénico?) de los profesionales}

Este punto intenta repensar el lugar del terapeuta.

La propia incapacidad que los pacientes manifiestan para superar su malestar puede transferirse sobre el terapeuta para con su labor de ayudarles a mejorar. Las consultas suelen desarrollarse con un discurso defensivo del paciente, que está pegado a su sintomatología. Los terapeutas (según los paradigmas teóricos de referencia que cada uno tuviera) en el intento de transformar la sesión en algo más que una revisión de síntomas y hacer progresar la psicoterapia, invitamos al paciente a hacer pasajes por sus experiencias vitales, rastreamos su psicobiografía, realizamos señalamientos hacia su pasado, analizamos las relaciones que mantuvieron con las figuras originarias o que mantienen con sus vínculos más recientes, modificamos o ajustamos sus tratamientos farmacológicos, aplicamos técnicas psicológicas variadas, etc. Este trabajo no tiene más validez que la de su propia duración y en cuanto acaba la sesión termina todo. En la siguiente sesión todo volverá a ser igual. Así, las sesiones tienen que repetirse indefinidamente.

Cabe reflexionar por nuestra parte si a los pacientes que, año tras año, presentan una posición distímica en consulta los estamos reforzando de un modo iatrogénico en sus mecanismos patógenos, cabe pensar si estamos cometiendo un error técnico al prolongarles indefinidamente los tratamientos ofertándoles indiscriminadamente cita tras cita.

Los pacientes parecen haber proyectado las partes más sanas y maduras de su self y conservan para sí los aspectos más enfermos e inmaduros. Si estos aspectos enfermos los reconocemos y validamos en exceso, estaremos estimulando continuamente las tendencias regresivas y de dependencia del paciente, a expensas de su autonomía y capacidad de crecimiento. Cualquier intento de trabajo terapéutico que ponga en juego el dilema autonomía-dependencia será vivido como una amenaza a la homeostasis y generará una agudización de los síntomas.

El terapeuta puede responder a la agudización de síntomas con un mayor arsenal terapéutico (aumentar la frecuencia de las visitas, aumentar o cambiar la me- 
ORIGINALES Y REVISIONES

dicación, pedir la colaboración de otros profesionales..). Este movimiento refuerza aún más la posición de enfermo en el paciente y la desresponsabilización del mismo sobre su cambio.

\section{¿Cómo explicar las resistencias al cambio?}

Este punto intenta conectar la teoría del desarrollo de la neurosis infantil (establecida en la relación con las figuras originarias de la infancia) con su reedición en la neurosis de transferencia (establecida con el terapeuta). Para ello tomamos como referencia el texto de Freud "Más Allá del Principio del Placer" (1).

Nos atreveremos a hacer una hipótesis de las ecuaciones inconscientes reprimidas que operan en el paciente y que se manifiestan en la transferencia. Curación = separación del terapeuta. Separación del terapeuta $=$ angustia/displacer. Enfermedad $=$ Amor $\mathrm{y}$ atención del terapeuta.

Freud (1) dice que el florecimiento temprano de la vida sexual estaba destinado a fracasar porque los deseos del infante eran inconciliables con la realidad y por la insuficiencia de la etapa evolutiva en la que se encontraba el niño.

Textualmente añade: "el vínculo tierno establecido casi siempre con el progenitor de sexo opuesto sucumbió al desengaño, a la vana espera de una satisfacción, a los celos que provocó el nacimiento de un hermanito, prueba indubitable de la infidelidad de la amada o del amado" y más adelante "el retiro de la ternura que se prodigaba al niñito, la exigencia creciente de la educación, palabras serias y un ocasional castigo habían terminado por revelar al niño todo el alcance del desaire que le reservaban. Así llega a su fin el amor típico de la infancia y su ocaso responde a unos pocos tipos, que aparecen con regularidad" (1).

Se está hablando de un duelo por la pérdida de amor. Según Freud, este fracaso deja como secuela una falta permanente en el sentimiento de sí que posteriormente será un poderoso aporte al sentimiento de inferioridad de los neuróticos.

Ahora bien, señala (1), "los neuróticos repiten en la transferencia todas estas ocasiones indeseadas y estas situaciones afectivas dolorosas, reanimándolas con gran habilidad. Saben procurarse de nuevo la impresión del desaire, fuerzan al médico a dirigirles palabras duras y a conducirse fríamente con ellos"

Se trata, entonces, de la acción de pulsiones que estaban destinadas a conducir a la satisfacción; pero ya en aquel momento no la produjeron, sino que conllevaron únicamente displacer. "La experiencia fue en vano pero se la repite a pesar de todo; una compulsión esfuerza a ello. La compulsión a la repetición es ganada para el yo, que quiere aferrarse al principio de placer". 


\section{Los límites al tratamiento} lógicos.

Este punto intenta justificar la necesidad de poner fin a los tratamientos psico-

En el texto Análisis Terminable e Interminable (1937), correspondiente al final de su obra, Freud (2) deja un claro poso de pesimismo acerca de la eficacia de los tratamientos psicoanalíticos. Considera continuamente los límites de la tarea terapéutica mencionando los aspectos inmanentes, biológicos e irreductibles del padecer del ser humano, el aspecto cuantitativo referido a la intensidad de la pulsión, los obstáculos para tratar los trastornos de etiología no traumática (se referiría con "no traumática" a todos aquellos que no son de tipo adaptativo), el arraigo de los comportamientos patógenos de los pacientes y su resistencia a la curación.

Freud (2) plantea como deseable los intentos de abreviar los tratamientos y él mismo, para un buen número de casos, utiliza la fijación de un plazo. Respecto del plazo, alerta sobre los riesgos de que el acortamiento de los tratamientos derive de un intento desafortunado de ajustar el tempo requerido para el análisis a las prisas de la vida contemporánea. En los tiempos de hoy en día el riesgo puede venir desde las presiones externas institucionales que amenazan con burocratizar la práctica clínica. También señala que el analista debe haber sido analizado para no ser estorbado en su trabajo por sus propios defectos o puntos ciegos.

Las condiciones que Freud (2) sugiere para el final de un análisis son: que el paciente no padezca a causa de sus síntomas, que haya superado sus angustias y que el analista juzgue haber hecho consciente tanto de lo reprimido, esclarecido tanto de lo incomprensible o eliminado tanto de la resistencia interna para que no se repitan los procesos patógenos en cuestión.

Para determinados casos como los que nos ocupan en este artículo estas condiciones que acabamos de referir no se cumplen o se cumplen muy precariamente aunque no por ello pensamos que se deslegitime el fin del tratamiento...

\section{Un comentario sobre "Técnica Activa"}

Este punto describe lo que Ferenczi (3-4) llamó Técnica Activa e intenta abordar la cuestión de si es relevante y útil realizar variaciones de la técnica en beneficio del avance del tratamiento.

Ferenczi se empieza a plantear su "técnica activa" en algunos casos de histeria de angustia. Estos pacientes, a pesar del riguroso seguimiento de la asociación libre y su conocimiento de los complejos inconscientes, no conseguían superar algunos puntos muertos del análisis hasta que no se les incitaba a salir del seguro refugio que 
ORIGINALES Y REVISIONES

constituía su fobia y a exponerse, a título de ensayo, a la situación de la que habían huido con angustia debido a su carácter penoso.

A este procedimiento, que hoy en día podríamos redenominar de otras maneras, lo llamó "técnica activa", lo cual significa una intervención activa tanto por parte del médico como por parte del paciente, al que se le imponía una tarea particular. En los casos de fobia esta tarea consistía en realizar determinadas acciones desagradables.

Ferenczi (3-4) dice que este artificio puede ser perjudicial al inicio de la terapia porque estropea las tentativas de transferencia espontáneas pero que durante el proceso terapéutico puede ser beneficioso o incluso inevitable. Advierte que esta técnica es un arma de doble filo ya que trabaja "a contrapelo", es decir, en contra del principio del placer y recomienda que para ser aplicada haya indicios ciertos de la solidez de la transferencia.

Hacia el fin del tratamiento las condiciones varían y el analista ya no teme que el paciente huya. Es habitual, según Ferenczi, que haya incluso que luchar contra la tendencia del paciente a prolongar indefinidamente la cura, o sea, aferrarse a él en lugar de volver a la realidad. En los tratamientos es raro llegar al final sin intervenciones activas o consignas que el paciente deba cumplir. Ejemplo de lo mismo puede ser establecer una fecha final del tratamiento o empujar al paciente a tomar una decisión visiblemente madura ya, pero diferida por resistencia.

Volvemos a nuestros pacientes con distimia crónica. Les vamos a proponer como única alternativa terapéutica una terapia de grupo de periodicidad limitada y orientada al fin del tratamiento.

\section{La propuesta grupal}

Este punto trata de justificar el tratamiento en grupo para este conjunto de pacientes que estamos describiendo desde el inicio del artículo

Nuestros pacientes están habituados a prolongados seguimientos individuales. Entonces, es comprensible que el tipo de psicoterapia que les vamos a proponer les pueda generar zozobra. Inicialmente suelen rechazarla debido a que rompe la instalada relación dual (incestuosa) con el terapeuta individual.

Recordemos algunas de las palabras de Freud cuando explicábamos el origen de los comportamientos neuróticos "el retiro de la ternura que se prodigaba al niñito... el desaire... el sentimiento de falta..". A estos pacientes, la idea de retirarles la habitual consulta individual (exclusiva para ellos) y de que compartan en grupo sus vivencias y experiencias, les moviliza y descentra. ¡Vamos a hablarles de que habrá un destete!, i...de que existe un límite!. Visto así, el grupo representa la intromisión de un tercero indeseable. 
El grupo tendrá una periodicidad limitada y no podremos titubear acerca del hecho de que habrá un final, independientemente de los avatares con que hasta allí se llegue.

\section{Diseño de los grupos de alta}

Este punto describe el encuadre de los grupos realizados y las características clínicas y sociodemográficas de los componentes

\section{Encuadre}

Entre octubre de 2010 y junio de 2013 se han llevado a cabo sucesivamente 3 grupos de alta, que denominaremos grupo A (en adelante G-A, compuesto por 12 pacientes), grupo $\mathrm{B}$ (en adelante $\mathrm{G}-\mathrm{B}$, compuesto por 11 pacientes) y grupo $\mathrm{C}$, (en adelante G-C, compuesto por 12 pacientes) (siendo A el primer grupo realizado y $\mathrm{C}$, el último grupo realizado).

Los grupos tuvieron una duración de 8 meses el grupo A, 8 meses el grupo B y 6 meses el grupo C, recibiendo respectivamente un total de 16, 16 y 11 sesiones. Las sesiones fueron quincenales, de una hora y media de duración y se han desarrollado en la sala de grupos de la Unidad de Salud Mental Comunitaria Oriente, perteneciente a la Unidad de Gestión Clínica de Salud Mental del Hospital Virgen del Rocío de Sevilla.

Los grupos han estado abiertos a incluir pacientes cuyos facultativos referentes eran los distintos Psicólogos Clínicos y Psiquiatras de la Unidad. En caso de inclusión de pacientes de otros facultativos que no eran el terapeuta grupal, se les pedía a éstos que dieran por finalizado el tratamiento individual con el paciente y les dieran paso para recorrer la fase final de tipo grupal. Los límites del tratamiento pasan por dar por concluida cualquier otro tipo de atención que hubiera tenido hasta el momento el paciente. Este paso requirió de la coordinación entre profesionales (entre el facultativo referente y el terapeuta grupal).

Los pacientes se incorporan al grupo previa entrevista individual con el terapeuta grupal. La estructura del grupo es cerrada y tras las dos primeras sesiones no hay incorporación de nuevos miembros.

A los componentes del grupo se les explican los motivos de haber sido propuestos para el grupo, los objetivos que se plantean y los otros aspectos habituales del encuadre (compromiso de asistencia, puntualidad, confidencialidad, etc.). En la/s 
ORIGINALES Y REVISIONES

entrevista/s previa/s de valoración de inclusión en el grupo compartimos con el paciente la frustración y las limitaciones e insuficiencias con las que se ha saldado el proceso terapéutico de tantos años. Sin abrir nuevas grandes expectativas acerca de la última fase del tratamiento, podremos animar al paciente a poder beneficiarse del mismo en una diferente oportunidad (colectiva, grupal) que pueda ayudarle a autopercibirse de una manera más saludable, a fortalecerse en su capacidad de crecimiento y autonomía personal y a prepararse para el alta de la Unidad de Salud Mental.

\section{Características clínicas}

Un total de 35 pacientes diagnosticados de distimia (según la CIE-10) han sido convocados para los grupos (G-A: 12 pacientes; G-B:11 pacientes; G-C: 12 pacientes).

El tiempo medio de tratamiento individual de estos pacientes, previo a su inclusión en el grupo era de 4'7 años (5'7 años de media en los pacientes del G-A; 6'1 años de media en los del G-B y 2'5 años de media en los del G-C)

\section{Características sociodemográficas}

Estos grupos han sido compuestos por 21 mujeres y 14 hombres (G-A: 8 mujeres/4 hombres; G-B: 5 mujeres/6 hombres; G-C: 8 mujeres/4 hombres).

La edad media era de 54 años (G-A: 51 años; G-B: 56 años; G-C: 55 años). La distribución según edades era: menores de 40 años: 1 paciente; entre 40 y 50 años: 9 pacientes; entre 51 y 60 años: 19 pacientes; mayores de 61: 2 pacientes.

Respecto al estado civil, tenemos un total de 28 casado/as, 3 viudo/as, 2 separado/as o divorciado/as y 2 soltero/as, siendo esta proporción según los grupos $\mathrm{A}, \mathrm{B}$ y C, y respectivamente, A:10,2,0,0; B:7,1,1,2; C:11,0,1,0.

La situación laboral de los componentes de los grupos era de 4 activo/as laboralmente y 31 inactivo/as ( 8 por desempleo, 7 por baja laboral y 16 por incapacidad). La proporción según los grupos A, B y C, y respectivamente, A:1,4,3,4; B:1,2,2,6; $\mathrm{C}: 2,2,2,6$.

La autopercepción que tenían de su grado de funcionamiento social fuera del entorno familiar era nula o escasa en 26 pacientes y aceptable en 9 pacientes. La proporción según los grupos A, B y C, y respectivamente, A:10,2; B:9,2; C:7,5.

El desarrollo de actividades promotoras de salud era nulo o escaso en 27 pacientes y aceptable en 8 pacientes. La proporción según los grupos $\mathrm{A}, \mathrm{B}$ y $\mathrm{C}$, y respectivamente, A: 12,0; B:8,3; C:7,5. 


\section{El desarrollo del grupo}

Este punto relata algunas impresiones del terapeuta acerca del proceso grupal seguido por los pacientes.

Fase inicial

Cuando en las entrevistas pregrupo se le explica a los pacientes que a la finalización del grupo recibirán el alta de las consultas, suelen quedar desconcertados. La curación es vista como un peligro porque supone la separación. El paciente habrá de enfrentarse a una situación nueva que en principio puede calificar como inútil o sentir que le hará empeorar ya que tiende a imaginarse un escenario rodeado de personas extrañas llenas de penas y de problemas que le serán contagiados.

Hubo 6 pacientes que decidieron no iniciar el grupo a pesar de que era la única alternativa terapéutica que se les ofertó. No estaban dispuestos a poner en cuestión su forma de pulsión, entendida ésta como un modo tozudo, utópico, inconsciente de intentar recuperar un paraíso perdido .

Un fenómeno grupal habitual en el trascurso de las sesiones iniciales es el ataque al grupo (desprestigiando el tratamiento compartido). Así, se pueden escuchar frases como "el grupo no me sirve para nada".

Puede que hasta bien avanzadas las sesiones no retengan el nombre de sus compañeros y que funcionen en el espacio vincular a modo de islotes o con las reminiscencias del tratamiento individual, dirigiéndose al terapeuta a modo de consultas individuales. El terapeuta se esforzará por señalar los mecanismos de escisión y aislamiento para que el grupo progrese como un todo

Fase intermedia

El avance del grupo hará que, por lo general, transformen la ansiógena percepción inicial por otra más confortable, en la que pueden sentir el hecho de que compartir con los demás las experiencias les sirve de desahogo de la tristeza interior, les saca del aislamiento mitigando sus síntomas depresivos y, en los casos que mejor evolucionan, tal vez les sirve de pre(lanzamiento) para un modo de vida más socializado.

El grupo en determinados momentos puede funcionar de un modo más constructivo, intentando superar sus limitaciones en una tarea compartida. Cito algunas frases que sugieren un cambio: "Antes pensaba que lo mío era lo peor del mundo pero me doy cuenta de que hay gente peor que yo" "me he dado cuenta de que tengo que aceptar la realidad y vivir lo mejor posible".

Fase final

El fantasma del corte final se manifiesta transversalmente a lo largo de todos los meses que dura el grupo y lo hace de modo especial en las últimas sesiones. Es frecuente que los pacientes añoren su vida personal pasada, siempre más satisfactoria que todo lo que les rodea en el presente, y que expresen su deseo fantasioso de recuperarla. También que se vuelvan a sentir impotentes para el cambio y para manejar sus vidas. 
Si pensamos en el grupo de alta como un grupo de duelo es natural observar (casi siempre de forma desplazada) una predominancia de sentimientos de desvalimiento, abandono, rabia, soledad, impotencia.

El tratamiento grupal les convoca con su "obligación" de responsabilizarse para buscar nuevos modos de satisfacción en sus vidas, nuevas alternativas.

El final del tratamiento incluye la entrega a cada paciente de un informe de alta de las consultas de Salud Mental. Al alta, pedimos a los componentes del grupo que prueben a realizar algunos cambios en su forma de vida y a compaginarlos con una progresiva desmedicalización de sus síntomas. Esta tarea la pueden hacer en colaboración con sus médicos de atención primaria En el informe se indica las recomendaciones terapéuticas a seguir por el paciente; éstas pueden incluir el cumplimiento de medidas higiénicas saludables de tipo lúdico, ocupacional, social, ejercicio físico, etc

El informe de alta al paciente oficializa el cierre del proceso en Salud Mental.

\section{Resultados}

Este punto da cuenta de los resultados obtenidos en los grupos A, B y C respecto a: 1- la asistencia media a las sesiones grupales.

2- al tipo de alta que reciben (alta antes de iniciar el grupo por rehusar el tratamiento, alta prematura por abandono del grupo o alta a la finalización del grupo)

3- al motivo de las altas prematuras (durante el proceso grupal)

4- al mantenimiento del alta transcurridos 3 años, 2 años o 1 año

5- al uso de tratamiento psicofarmacológico tomando como puntos de referencia el momento del alta y transcurridos 3, 2 o 1 año del alta (según el grupo)

\section{1- Respecto a la asistencia a las sesiones grupales:}

Si tenemos en cuenta los resultados de los tres grupos, obtenemos que asisten de promedio al $71^{\prime} 2 \%$ de las sesiones grupales.

En el grupo A hay una media de asistencia a 11'6 sesiones sobre 16, representa una asistencia media de los miembros del $72{ }^{\prime} \%$.

En el grupo B hay una media de asistencia a 11 sesiones sobre 16; representa una asistencia media de los miembros del $68,7 \%$.

En el grupo $\mathrm{C}$ hay una media de asistencia a 8 sesiones sobre 11, representa una asistencia media de los miembros del $722^{\prime} 7 \%$.

2-Respecto a los tres tipos de alta que reciben (alta antes de iniciar el grupo por rehusar el tratamiento, alta prematura por abandono o alta a la finalización del grupo)

De los 35 pacientes propuestos para los tres grupos obtenemos que 6 reciben el alta por rehusar el tratamiento grupal, 9 reciben el alta prematura (antes de acabar el grupo) y 20 reciben el alta al finalizar el grupo: 
En el grupo A (12 pacientes): 2 altas por rehusar el tratamiento, 2 altas prematuras y 8 altas al finalizar el grupo

En el grupo B (11 pacientes): 1 alta por rehusar el tratamiento, 5 altas prematuras y 5 altas al finalizar el grupo

En el grupo A (12 pacientes): 3 altas por rehusar el tratamiento, 2 altas prematuras y 7 altas al finalizar el grupo

3- Respecto a los motivos de las altas durante el proceso grupal (altas prematuras) obtenemos:

Grupo A: 2 pacientes (uno por mejoría y otro por cambio de facultativo)

Grupo B: 5 pacientes (uno por trabajo, otro por mejoría, 3 por alta voluntaria)

Grupo C: 2 pacientes (uno por mejoría y otro por intervención quirúrgica)

Por mejoría indica que el paciente decide dejar el grupo por iniciativa propia y hablándolo con los demás componentes debido a que se encuentra mejor y decide no llegar al final. Por alta voluntaria indica que el paciente deja de acudir a las sesiones y al llamarle por teléfono para preguntar el motivo dice que no quiere continuar sin expresar que haya habido una mejoría o un empeoramiento. Cambio de facultativo indica que la paciente no está conforme con que vaya a recibir el alta y opta por pedir un cambio de facultativo antes de que finalice el grupo, por lo que reinicia proceso individual con otro profesional de la Unidad. Por trabajo indica que la paciente ha encontrado un trabajo incompatible con el horario de las sesiones. Por intervención quirúrgica indica que el paciente no puede continuar con las sesiones por impedimento físico.

4- Respecto al mantenimiento del alta transcurridos 3 años, 2 años $o 1$ año según el grupo A, B o C

Resultados después de transcurridos 3 años desde el final del tratamiento del grupo A

Eran 12 pacientes, de los cuales, 9 no volvieron a reconsultar en la Unidad de Salud Mental, 1 consultó dos veces mas de forma individual y recibió el alta sin volver a reconsultar después y 2 siguen consultando de forma individual en la actualidad.

Resultados después de transcurridos 2 años desde el final del tratamiento del grupo B

Eran 11 pacientes, de los cuales, 8 no volvieron a reconsultar en la Unidad de Salud Mental y 3 reconsultaron dos veces mas de forma individual y recibieron el alta sin volver a reconsultar después.

Resultados después de transcurrido 1 año desde el final del tratamiento del grupo C. Eran 12 pacientes, de los cuales, 11 no volvieron a reconsultar en la Unidad de Salud Mental y 1 sigue consultando de forma individual en la actualidad.

En suma, del total de 35 pacientes distribuidos entre los tres grupos, 32 siguen de alta transcurridos 3 años, 2 años o 1 año a la finalización de los respectivos grupos. De los 32 que siguen de alta, 28 no volvieron a reconsultar tras finalizar el grupo y 
4 volvieron a reconsultar pero tras dos nuevas consultas individuales no volvieron a reconsultar. 3 pacientes reconsultaron y en la actualidad siguen siendo atendidos en la Unidad de Salud Mental

5- Respecto al uso de tratamiento psicofarmacológico tomando como puntos de referencia el momento del alta y transcurridos 3, 2 o 1 año según el grupo $\mathrm{A}, \mathrm{B}_{\mathrm{o}} \mathrm{C}^{\mathrm{1}^{*}}$ En el grupo A: 11 pacientes de 12 tenía al menos un antidepresivo y un ansiolítico al recibir el alta. Transcurridos 3 años del alta, 4 de ellos no tiene tratamiento farmacológico y 7 mantienen el que tenían al alta (o similar- a veces otro antidepresivo distinto).

En el grupo B los 11 pacientes tenían al menos un antidepresivo y un ansiolítico. Transcurridos dos años del alta, 3 de ellos no tienen tratamiento farmacológico y 2 han reducido las dosis de antidepresivos. El resto (6) mantienen el mismo tipo de tratamiento.

En el grupo C, 11 de 12 pacientes tenían algún tipo de psicofármaco prescrito. 8 pacientes tenían al menos 1 antidepresivo y 3 pacientes tenían sólo algún ansiolítico. Transcurrido un año del alta, 4 pacientes no tienen tratamiento farmacológico, 2 han reducido las dosis de ansiolíticos y uno las ha aumentado.

En suma, de los 35 pacientes propuestos para los grupos, 2 nunca tuvieron prescritos psicofármacos. De los 33 que tenían prescritos psicofármacos al alta, 11 no tienen tratamiento psicofarmacológico en la actualidad y 22 mantienen el mismo tratamiento farmacológico o similar.

\section{Comentario de los resultados obtenidos}

En Análisis Terminable e Interminable (2), Freud hace referencia a que a menudo se escucha decir a algunos terapeutas, a modo de lamento o de disculpa, que el análisis del paciente no fue terminado o que el paciente no fue analizado hasta el final. Reconocemos así en nuestra labor de terapeuta y en las palabras de despedida de los pacientes que se van de alta, ese sabor amargo, esa frustración propia del que tan solo ha conseguido, tras un seguimiento de años y años, un estado de normalidad psíquica parcial.

Pensamos que en nuestros pacientes sí pudieron observarse ciertos cambios cuantitativos, en la magnitud del sufrimiento, pero que los cambios cualitativos no siempre se producen. Freud explicaría este fenómeno diciendo que "el gobierno sobre lo pulsio-

\footnotetext{
${ }^{*}$ Hemos obtenido este dato no por la información directa del paciente sino mediante la consulta en la historia clínica digital del usuario (Diraya), donde figuran actualizados todos los tratamientos farmacológicos que sigue un paciente.
} 
nal mejora pero sigue siendo incompleto porque la transmudación del mecanismo de defensa ha sido imperfecta. Nada hay de asombroso en ello pues el análisis no trabaja con recursos ilimitados sino restringidos y el resultado final depende siempre de la proporción relativa entre las fuerzas de las instancias en recíproca lucha"

Los objetivos del grupo de alta eran mejorar a los pacientes en su autoeficacia percibida, fomentar su potencial de autonomía y prepararles para el alta de la Unidad de Salud Mental. No podemos aventurarnos a decir que los objetivos propuestos se hayan conseguido o al menos no hemos alcanzado a observarlos al final del grupo (en las sesiones finales de despedida)

Una de las hipótesis que barajamos es que el trabajo terapéutico pudiera empezar a notarse en el momento en que el paciente deja de tener citas en la Unidad. Dejará de tener el sostén fantaseado del terapeuta.

Pero ¿dejará de buscar el paraíso de un tiempo pasado que siempre fue mejor que el del presente?. Quizás, al verse convocado por el principio de realidad (sin tener la prótesis artificiosa del terapeuta para su malestar) tendrá que hacer frente a su vida con nuevas formas sublimatorias de la pulsión.

Los resultados obtenidos (respecto a que transcurridos 3, 2 y 1 años del alta en los diferentes grupos, 32 de los 35 pacientes convocados siguen de alta) nos hacen pensar con cierto optimismo en que los pacientes hayan podido transmudar algo de su posición distímica.

Si el trabajo terapéutico hubiera sido estéril, la insistencia pulsional autodestructiva operante en estos pacientes hubiera seguido viva, y es probable que muchos de ellos hubieran buscado nuevamente el canal para aferrarse al seguimiento en la Unidad. Esto no hubiera sido una tarea difícil de conseguir para el paciente ya que pueden pedir un cambio de facultativo que les permite una reevaluación de su caso por otro profesional o entrar de nuevo en las consultas por vía de rederivación desde atención primaria. Solamente una paciente pidió cambio de facultativo, además de otros dos pacientes que siguen siendo atendidos en la Unidad.

\section{Otras preguntas}

En este apartado formulamos una serie de interrogantes que para con nuestra labor clínica se desprenden de este trabajo

Sobre el alta del paciente

¿Cuál es el límite del tratamiento de un paciente? ¿hasta donde hemos de llegar con el paciente en la propuesta puramente elaborativa de los conflictos? ¿cuál es el papel que debe jugar la técnica activa a la hora de hacer avanzar un tratamiento estancado? ¿se debe resolver el alta a un paciente que tenga síntomas? ¿qué balance de riesgos-beneficios puede tener el final del tratamiento? 
ORIGINALES Y REVISIONES

\section{Sobre la psicoterapia grupal}

Aun siendo creyentes de que una psicoterapia de grupo por sí misma tiene una gran potencia terapéutica, podemos cuestionarnos si ¿es necesario el tratamiento grupal de este perfil de pacientes o bastaría con que cada terapeuta individual tuviera en el horizonte los límites de un tratamiento para así podérselo trasmitir al paciente en el momento preciso? ¿el grupo es un valor añadido a la última fase del tratamiento de estos pacientes?

¿qué multiplicidad de representaciones le vamos atribuyendo al grupo durante su desarrollo: representa un objeto transicional hacia el alta, una forma de alivio compartido, una oportunidad, un límite, etc.?

¿Deberíamos utilizar sistemas de evaluación que incluyan al menos indicadores de proceso grupal? ¿ Serían de interés otro tipo de evaluaciones que incluyera indicadores individuales pre y/o post grupo mediante cuestionarios de expectativas, de sintomatología percibida o de la satisfacción del paciente? Tenemos resistencia a utilizar sistemas de evaluación convencionales.

\section{Sobre la técnica grupal}

La técnica que empleamos es de corte grupo psicodinámico basado en el aquí y ahora. Se toma al grupo como objeto principal para analizar las interacciones entre sus miembros, utilizando cualquier elemento grupal presente como potencial que favorecerá el aprendizaje emocional e interpersonal. Sobre esta base (cercana al pensamiento teórico de grupo operativo) no dejamos de plantearnos si tendrían interés introducir puntualmente cambios técnicos proactivos (psicodramáticos, sistémicos, etc.) para ayudar a movilizar las defensas del grupo y hacerlo avanzar.

Nos surge la cuestión en torno al grado de directividad adecuada para movilizar el grupo sin convertirnos una vez más en los solucionadores de sus problemas.

¿Sería interesante la heterogeneización de diferentes perfiles en la composición de los grupos?

\section{Sobre el post-alta}

El no saber el destino de los pacientes (más allá de que podamos averiguar si reconsultan en salud mental o de si su médico de atención primaria modifica su tratamiento farmacológico) nos deja con incógnitas ya planteadas.

¿los efectos del grupo terapéutico se dan a posteriori? Si hubiera cambios, ¿a qué nivel se darían? ¿habrá una modificación de los rígidos esquemas que operan en ellos habitualmente? ¿habrá una modificación de los estilos de vida? ¿todo seguirá igual?

¿sería interesante una cita individual pasado un año para conocer la situación del paciente o, por el contrario la perspectiva de esta cita dejaría al paciente estancado en su no cambio?

Si los pacientes continúan siendo atendidos por su médico de atención primaria ¿que tipo de depositaciones transferenciales realizaran sobre este profesional? 
Sobre el paciente y el sistema de atención de la Sanidad Pública

¿Existen aspectos no revelados por el paciente cuyo tratamiento se cronifica? ¿Podemos pensar si en determinados casos, a la neurosis del paciente, secundariamente validada por el entorno (médico, familiar) se le potencia aún más por una ganancia terciaria en forma de incapacidad laboral permanente (que se remunera económicamente y de por vida)? ¿Un paciente se cura en el momento en que consigue una pensión económica por su patología?

¿De qué cuestiones serían emergentes los pacientes con distimias resistentes en nuestro sistema de atención pública?

BIBLIOGRAFÍA

(1) Freud, S. Más Allá del Principio de Placer (1920). En Obras Completas (vol. XVIII). Buenos Aires: Amorrortu, 1992; p. 1-62.

(2) Freud, S. Análisis Terminable e Interminable (1937). En Obras Completas (vol. XXIII). Buenos Aires: Amorrortu, 1992; p. 211-254.

(3) Ferenczi, S. Prolongaciones de la técnica activa en psicoanálisis (1921). En Obras Completas (tomo III). Madrid: Espasa Calpe, 1981; p. 137-156.

(4) Ferenczi, S. Contraindicaciones de la técnica activa (1926). En Obras Completas (tomo III). Madrid: Espasa Calpe, 1981; p. 427-438. 